Universitas, Volumen 1, Número 2, 2008, 64-69

๑) 2008 UNAN-León, Editorial Universitaria

\title{
Evaluación de los aprendizajes de historia iberoamericana adquiridos por los estudiantes nicaragüenses de educación media en al año 2006
}

Yovani Valle ${ }^{1 *}$, Monika Strasser $^{2}$, Vinicio Sandino ${ }^{3}$

'Departamento de Ciencias Sociales; Facultad de Ciencias de la Educación y Humanidades;

${ }^{2}$ Centro de Estudios Históricos; Facultad de Ciencias de la Educación y Humanidades; ${ }^{3}$ Dirección de Análisis Institucional y Evaluación; Universidad Nacional Autónoma de Nicaragua, León (UNAN-León).

\section{RESUMEN}

En Nicaragua son pocos los estudios dedicados a investigar la enseñanza de la historia, lo que es sumamente preocupante porque sólo a través de la investigación se puede descender a las prácticas de la enseñanza de la historia para analizarlas, comprenderlas y mejorarlas. El objetivo de esta investigación, consiste en evaluar el aprendizaje de la historia iberoamericana en la educación secundaria. La importancia de la enseñanza de la historia iberoamericana ha sido agenda de discusión de la Organización de los Estados lberoamericanos para la Educación, la Ciencia y la Cultura (OEI) desde los años 90 del siglo pasado resultando en la creación de la Cátedra de Historia de Iberoamerica. El presente trabajo es una investigación explorativa, con enfoque cuantitativo basada en la aplicación de 469 encuestas a estudiantes de la secundaria de 14 colegios en 8 departamentos del país. Las preguntas del cuestionario se refieren a los conocimientos, las percepciones y las valoraciones de los estudiantes respecto a la historia iberoamericana. Los resultados revelan que la mayoría de los estudiantes tienen graves deficiencias en cuanto a los conocimientos sobre el pasado de su propio país y los demás países iberoamericanos. Respecto a las representaciones se nota que otorgan mucha importancia al actuar de personalidades como Colón, San Martín y Bolívar. Además se observa que ven como causa principal de la existencia de una historia común de los países latinoamericanos el antiimperialismo y la revalorización de la herencia indígena.

Palabras claves: Evaluación de aprendizaje de historia; Enseñanza de historia en Nicaragua; Historia iberoamericana

\section{INTRODUCCIÓN}

En Nicaragua, la investigación educativa, comparada con la que se realiza en otras áreas del conocimiento, es la menos desarrollada, lo que se refleja en sus bajos índices de publicaciones en revistas indexadas. $Y$ dentro de este campo, son pocos los estudios dedicados a investigar la enseñanza de la historia en la educación media.

EI Departamento de Ciencias Sociales de la Universidad Nacional Autónoma de Nicaragua (UNAN-León), consciente de la falta de oportunidad en el desarrollo de conocimientos, se sumó al proyecto internacional titulado "Representaciones y cambio de conceptos históricos. Estudio comparativo e implicaciones en la enseñanza de la Historia en América Latina y España", coordinado por la Universidad de Barcelona, España. Este proyecto pretende reflexionar sobre aspectos relacionados con la visión de la historia como materia escolar en diversos países del ámbito iberoamericano: Argentina, Brasil, Chile, España, México, Nicaragua y Venezuela.

La primera fase del proyecto, se dedica a evaluar el aprendizaje de la historia iberoamericana de los estudiantes de la educación media de Nicaragua. Las encuestas aplicadas a estudiantes de colegios públicos

\footnotetext{
*Autor para correspondencia: gvguadamuz@gmail.com
}

y privados en todo el país tienen dos enfoques: primero, la evaluación de los conocimientos adquiridos sobre la historia iberoamericana y segundo, el análisis de las representaciones sobre la historia iberoamericana.

La importancia del proceso enseñanza-aprendizaje de la historia iberoamericana ha sido reconocido por la Organización de los Estados Iberoamericanos para la Educación, la Ciencia y la Cultura (OEI), resultando en la creación de la Cátedra de Historia de Iberoamérica (1999) que pretende poner a disposición de los ministerios y secretarías de educación pública de todos los países iberoamericanos los instrumentos necesarios para facilitar la incorporación del estudio de los procesos históricos de los países iberoamericanos en los currículos de la enseñanza media o secundaria, proponiendo conceptos innovadores en el proceso enseñanza-aprendizaje..$^{[1]}$

El interés en la enseñanza de la historia de Iberoamérica está particularmente vinculado, con la idea de fomentar la solidaridad entre los países iberoamericanos a través de llevar adelante iniciativas que conduzcan a una visión compartida de la historia de Iberoamérica y su proyección en textos escolares. ${ }^{[2]}$

A pesar de que, la Organización de los Estados Iberoamericanos, se ha preocupado por la enseñanza de la historia iberoamericana, las autoridades educativas 
nacionales todavía no han reconocido la importancia de incorporar la esencia de las declaraciones de la OEI a partir de la creación de la cátedra. Como consecuencia, los currículos de historia de educación secundaria y superior desconocen un componente denominado "historia iberoamericana".

Así, se espera que los resultados de esta investigación serán tomados en cuenta por las autoridades educativas con el fin de adecuar los currículos en la educación media y superior nicaragüense para que la historia iberoamericana deje de ser enseñada de forma marginal dentro de otros componentes curriculares históricos.

\section{METODOLOGÍA}

Es una investigación explorativa de corte transversal con enfoque cuantitativo. Se aplicó un cuestionario a los estudiantes de educación media, para identificar el aprendizaje de la historia iberoamericana. El cuestionario está compuesto por los siguientes cuatro bloques:

Bloque 1: Visión y valoración de la ciencia de la historia $y$ de la historia escolar ${ }^{4}$

Bloque 2: Conocimientos básicos y representaciones sobre la historia común iberoamericana

Bloque 3: Conocimientos básicos y representaciones sobre la historia nacional

Bloque 4: Representaciones sobre las relaciones entre países de Iberoamérica

Para la evaluación de los aprendizajes, se conformó un test de conocimientos integrado por 22 preguntas, de selección múltiple y apareamiento, correspondientes a los bloques 2 y 3 . Para la evaluación de las representaciones (de los bloques 2, 3 y 4) se utilizaron preguntas basadas en la escala Lickert.

Los datos se capturaron en hojas de respuesta para su posterior lectura electrónica. El tratamiento estadístico se realizó usando el programa SPSS versión 12 ordenando los datos en tablas de frecuencia. La muestra es de 469 estudiantes de 14 centros de 8 departamentos del país. Donde fue posible la muestra departamental se conformó por un centro público y un centro privado.
Tabla 1. Números de estudiantes según centros educativos seleccionados

\begin{tabular}{|l|c|c|}
\hline \multicolumn{1}{|c|}{ Centros } & Frecuencia & Porcentaje \\
\hline $\begin{array}{l}\text { Colegio Mercantil de } \\
\text { Occidente, Chinandega }\end{array}$ & 33 & 7.0 \\
\hline $\begin{array}{l}\text { Colegio Rubén Darío, } \\
\text { Chinandega }\end{array}$ & 23 & 4.9 \\
\hline $\begin{array}{l}\text { Instituto Nacional de } \\
\text { Occidente, León }\end{array}$ & 35 & 7.5 \\
\hline La Asunción, León & 35 & 7.5 \\
\hline "Manuel Olivares", Managua & 35 & 7.5 \\
\hline "Guillermo Cano", Estelí & 35 & 7.5 \\
\hline $\begin{array}{l}\text { Instituto Nacional Francisco } \\
\text { Luis Espinoza, Estelí }\end{array}$ & 35 & 7.5 \\
\hline $\begin{array}{l}\text { Instituto Nacional de } \\
\text { Chontales, Chontales }\end{array}$ & 35 & 7.5 \\
\hline "Cristóbal Colón", Bluefields & 33 & 7.0 \\
\hline Moravo - Bluefields & 35 & 7.5 \\
\hline $\begin{array}{l}\text { Moravo - Juan Amos } \\
\text { Comenius, Puerto Cabezas }\end{array}$ & 35 & 7.5 \\
\hline Adventista, Puerto Cabezas & 34 & 7.2 \\
\hline Cristo Rey, Río San Juan & 34 & 7.2 \\
\hline $\begin{array}{l}\text { Instituto Carlos Borromeo, } \\
\text { Río San Juan }\end{array}$ & 32 & 6.8 \\
\hline Total & 469 & 100.0 \\
\hline
\end{tabular}

Tabla 2: Números de estudiantes encuestados por departamentos

\begin{tabular}{|l|c|c|}
\hline \multicolumn{1}{|c|}{ Departamentos } & Frecuencia & Porcentaje \\
\hline Chinandega & 56 & 11.9 \\
\hline León & 70 & 14.9 \\
\hline Managua & 35 & 7.5 \\
\hline Estelí & 70 & 14.9 \\
\hline Chontales & 35 & 7.5 \\
\hline RAAS & 68 & 14.5 \\
\hline RAAN & 69 & 14.7 \\
\hline Rio San Juan & 66 & 14.1 \\
\hline Total & 469 & 100.0 \\
\hline
\end{tabular}

Tabla 3: Distribución por Sexo

\begin{tabular}{|l|c|c|}
\hline \multicolumn{1}{|c|}{ Sexo } & Frecuencia & Porcentaje \\
\hline Masculino & 193 & 41.8 \\
\hline Femenino & 269 & 58.2 \\
\hline Total & 462 & 100.0 \\
\hline
\end{tabular}


Tabla 4. Nivel de Estudio

\begin{tabular}{|c|c|c|}
\hline Año & Frecuencia & Porcentaje \\
\hline III año & 13 & 2.8 \\
\hline IV año & 342 & 73.5 \\
\hline V año & 110 & 23.7 \\
\hline Total & 465 & 100.0 \\
\hline
\end{tabular}

\section{RESULTADOS}

\subsection{Datos generales}

En total, se logró encuestar a 469 estudiantes de 14 centros escolares (Tabla 1) en 8 departamentos (Tabla 2 ), de los cuales eran $41,8 \%$ masculino y $58,2 \%$ femenino (Tabla 3). Los estudiantes encuestados correspondían a $2,8 \%$ de III año, $73,5 \%$ de IV año y $23 \%$ de $\mathrm{V}$ año (Tabla 4 ).

\subsection{RENDIMIENTO ACADÉMICO}

La tabla 5 detalla, los resultados del test de conocimientos. El porcentaje se refiere a las repuestas correctas. Los resultados revelan que los estudiantes no tienen un buen dominio sobre la historia iberoamericana, con el porcentaje máximo $(60,6 \%)$ en la pregunta acerca de la civilización mexicana/mesoamericana.

En general, la tendencia es que los estudiantes tienen mejores conocimientos sobre la historia de América Central que del resto de lberoamérica (véase preguntas 5-10); así muy pocos estudiantes contestaron de forma correcta las preguntas 19-22, refiriéndose a aspectos históricos de Brasil, Paraguay, Chile y Argentina.

Sin embargo, lo que más llama la atención, es el desconocimiento de los estudiantes sobre personalidades importantes de la historia nacional. Así, el 54,9\% desconoce a Augusto César Sandino (Pregunta 8) y $50,9 \%$ a Bartolomé de las Casas (pregunta 7). El desconocimiento de Sandino, se puede atribuir a la poca importancia que le han otorgado a este héroe nacional en los programas de historia en la educación básica, media y superior a partir de 1990.

\subsection{REPRESENTACIONES SOBRE ASPECTOS DE LA HISTORIA IBEROAMERICANA}

Se seleccionaron las siguientes representaciones de los hechos históricos por el énfasis que tienen en el currículo de la educación media nicaragüense. Además, la mayoría de los libros de texto de Historia
Tabla 5. Test de conocimientos

\begin{tabular}{|l|c|}
\hline \multicolumn{1}{|c|}{ PREGUNTA } & $\%$ \\
\hline 1. Civilización de México (Mesoamérica) & 60.6 \\
\hline 2. Hugo Chávez - Presidente de Venezuela & 59.2 \\
\hline $\begin{array}{l}\text { 3. Eva Perón - Primera dama y lider de masas } \\
\text { Argentina }\end{array}$ & 57.7 \\
\hline 4. Atahualpa emperador Inca & 56.9 \\
\hline 5. Hernán Cortés Conquistador de México & 53.7 \\
\hline 6. Pueblos y Civilizaciones indígenas & 53.6 \\
\hline 7. Bartolomé de las Casas apóstol de los indios & 49.1 \\
\hline 8. Augusto Sandino - Guerrillero nicaragüense & 45.1 \\
\hline 9. Fecha de llegada de Colón & 43.7 \\
\hline 10. Civilización de Guatemala y Honduras & 43.5 \\
\hline 11. Civilización en Perú & 41.2 \\
\hline $\begin{array}{l}\text { 12. Zonas y países actuales del Virreinato de Nueva } \\
\text { España - México, América Central y Antillas }\end{array}$ & 39.1 \\
\hline 13. Pedro Alvarez de Cabral conquistador de Brasil & 33.0 \\
\hline 14. Emiliano Zapata - Líder agrario mexicano & 24.0 \\
\hline 15. Pueblos y civilizaciones con alto grado de poder & 23.7 \\
\hline 16. Francisco Franco - Dictador español & 20.1 \\
\hline $\begin{array}{l}\text { 17. Getulio Vargas - Líder de masas y presidente } \\
\text { brasileño }\end{array}$ & 18.8 \\
\hline $\begin{array}{l}\text { 18. Hechos relacionados al 10 de diciembre 1811 - } \\
\text { Conspiración de Belén }\end{array}$ & 16.0 \\
\hline 19. Civilización en Brasil y Paraguay & 11.8 \\
\hline 20. Salvador Allende - Presidente socialista chileno & 8.3 \\
\hline 21. Civilización en Chile & 2.3 \\
\hline 22. Civilización en Argentina (Patagonia) & 0.4 \\
\hline
\end{tabular}

de Nicaragua y América dedican suficiente espacio al desarrollo de estos temas.

\subsubsection{Causas y consecuencias de la llegada de los españoles al continente americano}

En la figura 1, se observa que los estudiantes ven como causas principales para explicar la llegada europea al continente americano el afán aventurero de Colón $(71,6 \%$ ) y la expansión demográfica $(69,4 \%)$. Se destaca que, las opciones seleccionadas remiten a una mezcla, tanto de necesidades particulares como sociales que habrían motivado la idea del descubrimiento de América.

La figura 2 detalla, las percepciones de los estudiantes acerca de las consecuencias de la llegada de los europeos al continente americano. El $73,0 \%$ considera que la consecuencia más importante era el crecimiento 
Figura 1. Causas de la llegada de los españoles

Por interés de la Iglesia Católica.

Por impulso económico de burguesia comercial.

Por interés de monarquia española.

Por expansión demográfica en el siglo XV.

Por afan aventurerio de Colón.

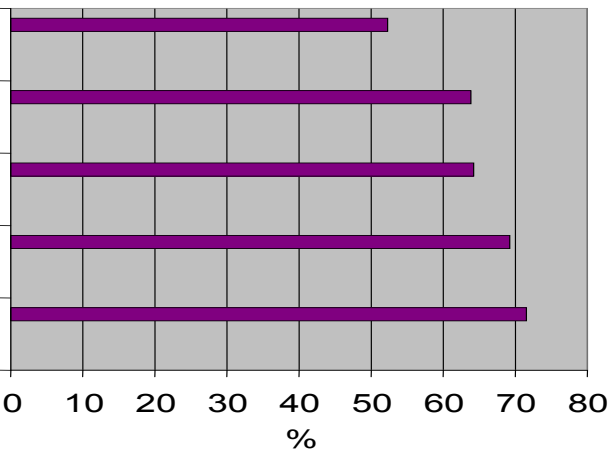

Figura 2. Consecuencias de la llegada de los españoles a América

Provocó igual desarrollo de Europa y los indígenas.

Provocó crecimiento y modernización de América.

Provocó crisis a lo largo del siglo XX.

Provocó eliminación física de indígenas.

Provocó crecimiento de economía europea.

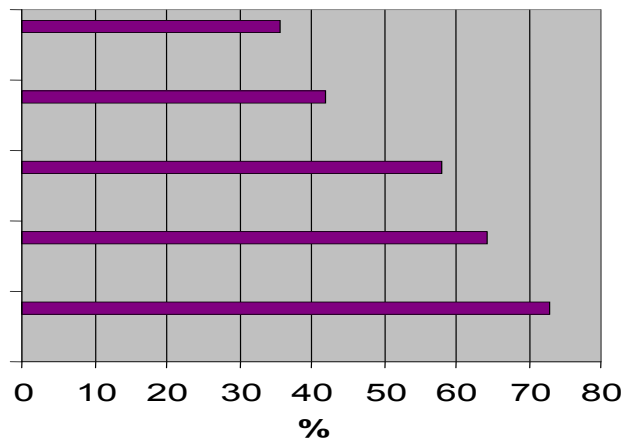

Figura 3. Causas de la independencia

Por crisis general de orden colonial en América por ineficiencia de reformas.

Por presión de élite criolla que buscaba acceder al gobierno.

Por crisis de imperio español por invasión napoleónica a España.

Por influencia de ideas liberales de revolución francesa e independencia de E.E.U.U.

Por acción de líderes como San Martín y Bolivar.

Por presión del pueblo americano.

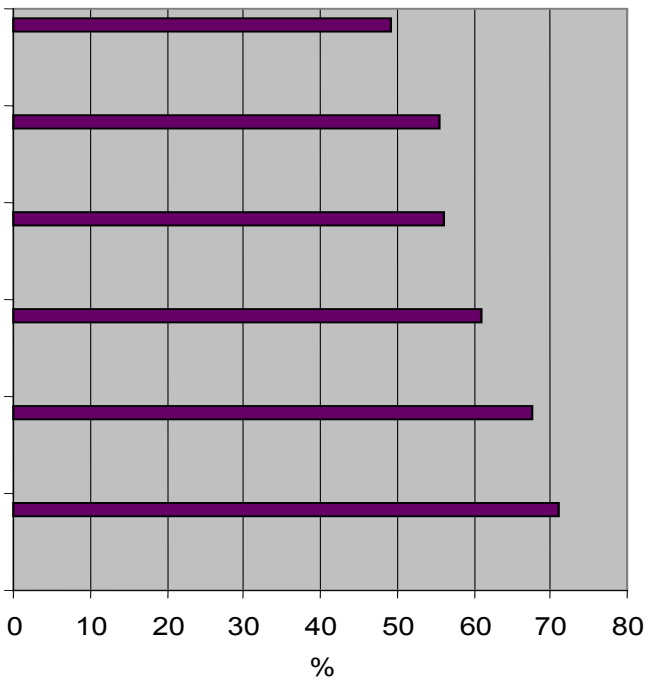

económico europeo, seguido por la eliminación física de indígenas $(64,3 \%)$. Paralelamente, sólo $35,7 \%$ opina que hubo igual desarrollo de Europa y los indígenas.

\subsubsection{Causas de la independencia}

La figura 3, evidencia que $71,2 \%$ de los estudiantes cree que la independencia se otorga principalmente al actuar del pueblo americano. Esto refleja el predominio del mito de su origen popular. Además, el resultado de esta pregunta indica una importante adhesión a aquella corriente de pensamiento que considera que la historia es determinada y construida por la acción de personajes puntuales (positivismo).

Esto se ve reflejado en el hecho que $67,6 \%$ de los estudiantes piensa que la independencia se logró a través de la actuación de líderes americanos como San Martín y Bolívar. 
Figura 4. Relaciones de Nicaragua con América Latina

Hay historia común por influencia española.

Hay pocos puntos en común por ser país pobre y mayor vínculo con E.E.U.U.

Hay historia común por influencia de pueblos indígenas.

Hay historia común por lucha contra fuerzas imperialistas.

Figura 5. Relaciones históricas con España

Hay que tener mucho cuidado con España por conflictos históricos que nos han perjudicado.

Hay que resguardar y valorar relación con España por ser país desarrollado y ha aportado mucho.

Hay que brindarle a España el mismo resguardo y valoración que a otro país.

Figura 6. Relaciones con Costa Rica

Hecho que mejor refleja actual situación con Costa Rica, es el apoyo brindado por Costa Rica en guerra contra Walker.

No se logrará ningún proyecto común con Costa Rica porque la economía costarricense ha sido más fuerte que la nicaragüense y lo es actualmente.

Hecho que mejor refleja actual situación con Costa Rica, es el conflicto por límites territoriales.

\subsection{VALORACIONES SOBRE RELACIONES ENTRE PAÍSES IBEROAMERICANOS}

En la figura 4 se observa que, los estudiantes ven como causa principal de la existencia de historia común entre Nicaragua y América Latina las luchas antiimperialistas $(70,7 \%)$, seguido por la importancia del papel de los indígenas $(63,1 \%)$.

La figura 5 detalla que, los estudiantes no tienen una percepción negativa de España; así el 77,4\% es de la opinión que hay que valorar las relaciones con España igual que con otros países.
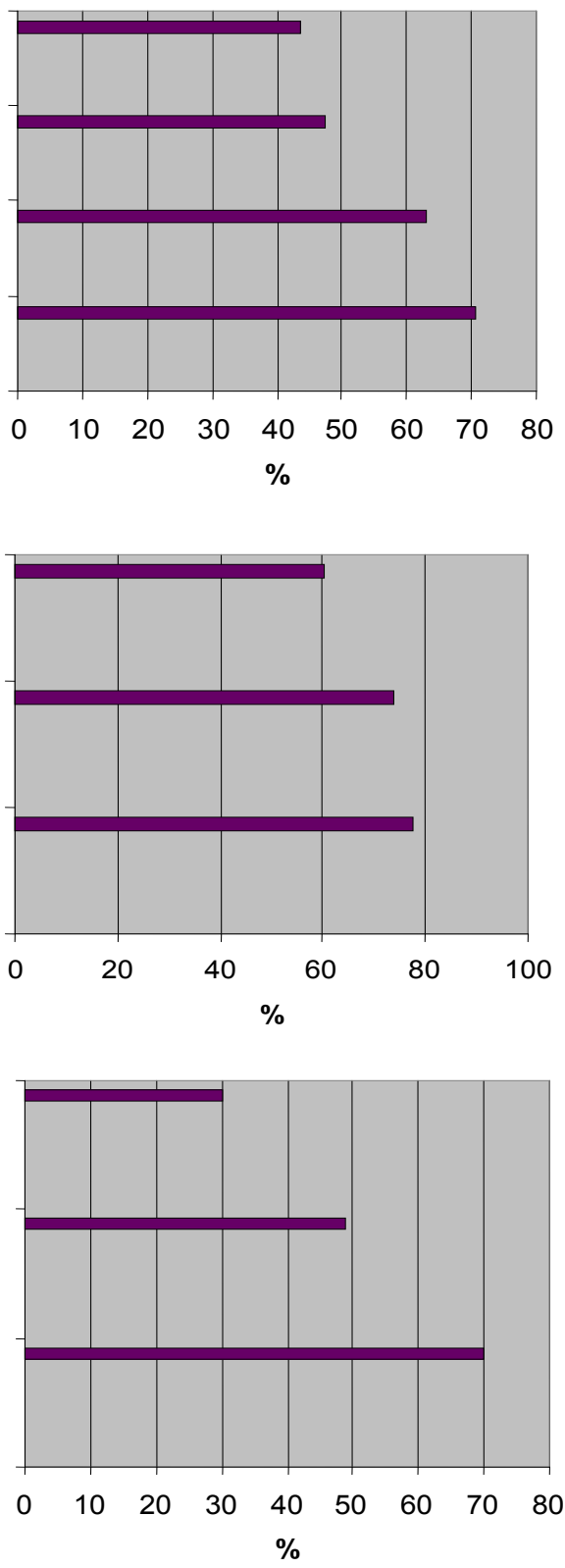

En las relaciones con Costa Rica destacan los conflictos limítrofes (figura 6). De esta manera $69,8 \%$ de los estudiantes opina que el hecho que mejor refleja la actual situación entre Nicaragua y Costa Rica son estos conflictos territoriales. Sin embargo, hay que reconocer que al momento de aplicar la encuesta (finales de 2006) este conflicto estaba en un punto alto.

\section{CONCLUSIONES}

En general, los estudiantes de la educación media nicaragüense tienen poco dominio sobre la historia iberoamericana. Se observaque, hay más conocimientos sobre aspectos relacionados con la historia de América 
Central que de América del Sur.

Resulta que, los estudiantes, desconocen sobre todo aspectos históricos relacionados con Argentina, Chile, Paraguay y Brasil. Sin embargo, también existen graves deficiencias acerca de la historia nacional manifestándose en el desconocimiento sobre personalidades clave de la historia nacional como Augusto César Sandino y Bartolomé de las Casas. Acerca de las representaciones se nota que, los estudiantes otorgan mucha importancia al actuar de personalidades, como Colón, San Martín y Bolívar, lo que refleja la visión positivista (enfoque en el estudio de la historia político-militar y grandes personajes) todavía predominante en el quehacer escolar y académico en torno a la historia en Nicaragua.

Además, la representación de las consecuencias de la colonización española valora más los aspectos negativos que los positivos (eliminación de los indígenas, crisis a lo largo del siglo XX).

Respecto a las valoraciones destaca que los estudiantes valoran el antiimperialismo como el elemento articulador de los países latinoamericanos junto con la revalorización de la herencia indígena. También se observa una valoración positiva de España. Sin embargo, las relaciones con Costa Rica siguen marcadas por los conflictos limítrofes.

\section{AGRADECIMIENTOS}

Al proyecto SEJ 2006-15461 financiado por la DGICT de la Universidad de Barcelona.

Al programa de "Pequeñas Ayudas de Investigación" financiadas por ASDI-SAREC de Suecia, UNAN-León.

A las autoridades y alumnos de centros educativos donde se realizaron las encuestas.

A los estudiantes participantes de Ciencias Sociales de la UNAN-León.

\section{REFERENCIAS}

1. Organización de los Estados Iberoamericanos. www.campusoei.org/cathistoria/presentación.htm; Acceso el 27 de octubre de 2007.

2. "Declaración de Viña del Mar, Chile, noviembre 1996" en: Ibidem. Acceso el 27 de octubre de 2007. 